\title{
PHYSICS OF THE L-MODE TO H-MODE TRANSITION IN TOKAMAKS
}

by

K.H. BURRELL, T.N. CARLSTROM, E.J. DOYLE, ${ }^{\dagger}$ D. FINKENTHAL, $\ddagger$

P. GOHIL, R.J. GROEBNER, D.L. HILLIS, J, KIM, H. MATSUMOTO, ${ }^{b}$ R.A. MOYER, ${ }^{\dagger}$ T.H. OSBORNE, C.L. RETTIG, ${ }^{\dagger}$ W.A. PEEBLES, ${ }^{\dagger}$ T.L. RHODES, ${ }^{\dagger}$ H. St. JOHN, R.D. STAMBAUGH, M.R. WADE, and J.G. WATKINS $\triangle$

This is a preprint of an invited peper to be presented at the Nineteenth European Conference on Controlled Fusion and Plasma Heating, June 29 through July 3. 1992, Innsbruck. Austria, and to be printed in the Proceedings.

Work supported by U.S. Department of Energy Contract DE-AC03-89ER51114

tUniversity of Califorria at Los Angeles.

‡ University of California at Berkeley.

"Oak Ridge National Laboratory.

bapan Atomic Energy Research institute.

$\triangle$ Sandia National Laboratories.

GENERAL ATOMICS PROJECT 3466 JULY 1992 


\title{
PHYSICS OF THE L-MODE TO H-MODE TRANSITION IN TOKAMAKS
}

K.H. Burrell, T.N. Carlstrorn, E.J. Doyle, ${ }^{1}$ D. Finkenthal, ${ }^{2}$ P. Gohil, R.J. Groebner, D.L. Hillis, ${ }^{3}$ J. Kim, H. Matsurnoto, ${ }^{4}$ R.A. Moyer, ${ }^{2}$, T.H. Osborne, C.L. Rettig, ${ }^{1}$ W.A. Peebles, ${ }^{1}$ T.L. Rhodes, ${ }^{1}$ H. StJohn, R.D. Stambaugh,M.R. Wade, ${ }^{3}$ and J.G. Watkins ${ }^{5}$

General Atomics, San Diego, California USA

${ }^{1}$ University of California at Los Angeles, USA

${ }^{2}$ University of California at Berkeley, USA

${ }^{3}$ Oak Ridge National Laboratory, USA

${ }^{4} J a p a n$ Atomic Energy Research Institute, JAPAN

${ }^{5}$ Sandia National Laboratories, Albuquerque USA

\begin{abstract}
Combined theoretical and experimental work has resulted in the creation of a paradigm which has allowed semi-quantitative understanding of the edge confinement improvement that occurs in the $H$-mode. Shear in the $E \times B$ flow of the fluctuations in the plasma edge can lead to decorrelation of the fluctuations, decreased radial correlation lengths and reduced turbulent transport. Changes in the radial electric field, the density fluctuations and the edge transport consistent with shear stabilization of turbulence have been seen in several tokamaks. The purpose of this paper is to discuss the most recent data in the light of the basic paradigm of electric field shear stabilization and to critically compare the experimental results with various theories.
\end{abstract}

\section{KEYWORDS}

Fusion; tokamak; confinement; divertor; H-mode; L-mode; L to H transition; DIII-D

\section{INTRODUCTION}

Understanding and improving energy confinement remains one of the arajor goals of the tokamak fusion program, since improved confinement can lead to lower development costs and a more econornically attractive fusion reactor. Since its original discovery in ASDEX (Wagner et al., 1982), the $\mathrm{H}$-mode has proven to be one of the most robust and ubiquitous of the improved confinement regimes seen over the last few years. Comparisons with other improved confinement regimes have shown the $\mathrm{H}$-mode to be the most reactor compatible (Wagner et al., 1991). Accordingly, to ensure that the $\mathrm{H}$-mode can be utilized in a reactor, it is important to understand how the H--mode develops out of the poorer confinement L--rnode. In addition, since there are indications that the same phenornena causing edge confinement improvement at the $L$ to $H$ transition may also lead to confinement improvement in the bulk of the discharge (Jackson et al., 1992; KurkjSuonio et al., 1992; Philipona et al., 1991a, 1991b), this understanding may also provide a route to further improvement in bulk confinement.

Over the last few years, combined theoretical and experimental work has resulted in the the creation of a paradigm which has allowed semi-quartitative understanding of the edge confinement improvement that occurs in the $\mathrm{H}$-mode. As has been discussed by Biglari etal., (1990) and Shaing et al., (1990), shear in the $E \times B$ flow of the fluctuations in the plasma edge can lead to decorrelation of the fluctuations, decreased radial correlation lengths and reduced turbulent transport. Behavior consistent with effects of shear in the radial electric field $E_{r}$ have been seen in several tokaialaks. At the $L$ to $H$ transition, the electric field stear develops in a localized region near the plasma edge (Doyle etal., 1991; Groebner et al., 1930a, 1991; Ida et al., 1990, 1992; Matsumoto etal., 1992; Van Nieuwenhove etal., 1991; Taylor et al., 1991). Turbulent density fluctuations decrease in the same region (Doyle et al., 1991; Groebner et al., 1991; Matsumoto et al., 1992; Tyman et al., 1992). Particle and energy transport decrease there also, as indicated by the increase in the local gradients (Doyle et ch., 1991; Gohil et al., 1991a; Groebner et al., 1991; Ida. 
et al, 1990, 1992; Tynan et al., 1992; Van Nieuwenhove et al., 1991) and by direct measurements of the local, fluctuation-driven particle flux (Moyer et al., 1992; Taylor et al., 1991: Tynan et al., 1992). In addition, as will be shown more clearly in the present work, a significant change in the electric field occurs right at the transition, before the radial profiles of density and temperature have had a chance to change appreciably (Doyle et al., 1991; Groebner et al., 1990a). This indicates that the initial ciange in $E_{r}$ is not caused by the improvement in confinement altering the radial profiles of density and temperature, although $E_{r}$ may change further while the profiles continue to evolve (Burrell et al., 1990; Field et al, 1991, 1992; Groebner et al., 1990a, 1992). Furthermore, the radial correlation length of the density fluctuations decreases from L-mode to H-mode (Rhodes et al., 15191; Taylor et al., 1991; Tynan, 1991), as is expected from theory (Biglari etal., 1990). Finally, information on the question of causality is also provided by experiments with $\mathrm{H}$-modes produced by direct biasing of the plasma in CCT (Taylor et al., 1989, 1991), TEXTOR (Weynants et al., 1991) and TUMAN3 (Askinazi etal., 1992). These show that altering the radial electric field causes the transition to occur. In addition to the data from $\mathrm{L}$ to $\mathrm{H}$ transitions, electric field shear stabilization effects have also been seen in the edge of Ohmically heated limiter discharges in TEX'T (Ritz et irl., 1990).

The purpose of this paper is to discuss the most recent data in the light of the basic paradigm of electric field shear stabilization, and to compare the experimental results with various theories which consider the effect of $E_{\tau}$ shear on the fluctuations or which attempt to explain how the sheared $E_{r}$ field is created.

\section{DEVELOPMENT OF THE BASIC PARADIGM}

in 1988, theoretical work by Itoh and Itoh $(1988,1989 \mathrm{a}, 1989 \mathrm{~b})$ and Shaing et al., (1989a), predicted that the radial electric field might play an important role in the physics of the $L$ to $H$ transition. (There had been previous deductions (Hinton, 1985; Ohkawa and Hinton, 1987) that a negative electric field would exist near but inside the separatrix.] Experimental work, which started independently about the same time, led later to publications which indeed showed the importance of the electric field (Burrell et al., 1989b; Groebner et al., 1989; Taylor et al., 1989), although some of the det ailed predictions of the original two theories were shown to require modification (Burrell et al., 1989b; Groebner et. al., 1990a, 1991).

A continuing experiment-theory interaction led to progress in both areas. Exposure of theorists to experimental results lead to further refinements in the theories (Itoh and toh, 1990; Shaing et al., 1989b, 1990), lucluding clarification of the bifurcation mechanism predictions, and the creation of new formulations of electric field shear stabilization (Biglari et al., 1990). In addition, competing models have been developed based on spontaneous poloidal spin-up (Hassam etal, 1991a), nonlinear transport equations (Hinton, 1991), and fluctuation-generated electric fields (Diamond and Kim, 1991). There has also been considerable theoretical discussion of whether linear stability calculations are sufficient (Hassam et al., 1991b; Staebler and Dominguex, 1991) or whether nonlinear effects will allow the plasma to still produce unstable modes even when the electric field shear is nominally sufficient to linearly stabilize the mode in question (Carrerus et al., 1992; Diamond and Kim, 1991).

Exposture to theories led experimentalists to improve the spatial resolution and tirne response of their rotation measurements (Field et al., 1991, 1992; Gohil et al., 1990, 1991b; Groebner et al., 1991; Hawkes et al., 1992; Yda and Hidekurna, 1989). In addition, greater focus on the detailed physics of the edge layer was given to the work with reflectometry (Doyle et al., 1990; Lehecka et al., 1988; Mansc etal., 1991), correlation reflectometry (Costley et al., 1990; Cripwell et al., 1989; Rhodes et al., 1992b), far infra-red (FIR) scattering (Holzhauer et al., 1990; Peebles et al., 1990; Philipona et al., 1990. 1991a, 1991b; Rettig et al., 1990) and Langmuir probes (Taylor et al., 1989, 1991; Tynan etal., 1991, 1992).

Because most early theory models were based on slab or cylinurical approximations, there initially was some confusion among experimentalists whether the shear in the poloictal rotation or the shear in the radial electric field was the irnportant quantity in stabilizing turbulence. (In slab and cylindrical geometries, the $E \times D$ flow is same as the poloidal ion mass flow.) In a torus, especially at low aspect ratio, the poloidal and $E_{r} \times B$ directions can be quite different (Groebner etal., 1990a). Work by Kirn et al., (1991a) makes clear that, for extended, flute-like modes, the $E \times B$ convection is the only ierm that appears in the stability equation. Measured parallel wavelengths for tokamak edge turbulence exceed poloidal wavelengths by more than a factor of 100 (Kim et al., 1991c), indicating that the assumption of flute-lise modes is reasonable. Accordingly, it is the shear in the $E_{r} \times B$ fow that is important in stabilizing turbulence. 


\section{EXPERIMENTAL RESULTS}

The latest results from DIII-D clearly show that the region just inside the separatrix where the electric field she ar develops is also the region where the density fluctuations decrease and where the tranoport improves. This behavior is illustrated in Fig. 1. In Fig. 1(a), $E_{r}$ and the poloidal rotation $v_{\theta}$ for $\mathrm{C}$ VII change right at the transition in a localized region just inside the sepaxarrix. The $E_{r}$ value is inferred from the radial force balance equation for C VII as discussed by (Groebner etal., 1991). Also shown in Fig. 1(a) is a curve showing the change in the density fluctuations as revealed by the reflectometer system. The locations of the reflectometer points are determined by using electron density profiles from the multipulse Thomson scattering system (Carlstrom etal., 1990). Although interpretation of reflectometer signals can have significant complications (Rhodes et al., 1992a), the plot in Fig. 1(a) indicates a significant change in the density fluctuations in the same narrow region inside the separatrix where the electric field shear develops. FIR scattering results (Philipona etal., 1991a, 1991b; Rettig et al. 1992a, 1992b) have confirmed that the density fluctuations just inside the separatrix decrease right at the $L$ to $H$ transition.

As is illustrated in Fig. 1(b) and (c), both the electron and ion particle and energy confinement improve in the region where the electric field shear is created and where the fluctuations decrease. Density and temperature profiles all steepen in this region after the transition, indicating a confinement improvement. In addition, initial measurements with a fast, moveable Langmuir probo system have shown a definite decrease in the fluctuation-driven particle flux at the separatrix in $\mathrm{H}$-mode relative to $\mathrm{L}$-mode (Moyer etal., 1992). This change in the fluctuation-driven flux is similar to that seen on CCT (Taylor et al., 1991; Tyman et al., 1992).

Although the DIII-D data are from the most complete set of measurements, results from several other machines are consistent with the picture of electric field shear stabilization. Measurements on JFT2-M (Ida etal., 1990, 199.2) have shown an increase in electric field shear and an increase in density and temperature gradients in the same region of the plasma after the $L$ to $H$ transition. The ASDEX group has reported a decrease in density fluctuations in $H$-mode as measured by reflectometry (Manso etal., 1991) and FIR scattering (Dodel etal., 1991, 1992) as well as some measurements detailing changes in the electric field (Field et al, 1991, 1992). Unfortunately, use of emission spectroscopy measurements along a single spatial chord did not permit (Field et al., $1991,1992)$ to determine the spatial structure of the radial electric field. A sheared electric field structure has also been seen in TEXTOR (Van Nieuwenhove et al., 1991) in an H-mode produced by directly biasing the plasma.

Not only is there a spatial correlation between increased shear in $E_{r}$, decreased density fluctuations and improved transport, there is also a temporal correlation. As is shown in Fig. 2 , on DIII-D, at the edge, the poloidal and toroidal rotation sperds of C VII, the inferred $E_{r}$ and the reflectometer fluctuation power all show dramatic changes at the time that the drop in the divertor $D_{\alpha}$ signal begins. TThe decrease in the $\mathrm{D}_{\alpha}$ signal is one of the most reliable indicators of the $\mathrm{L}$ to $\mathrm{H}$ transition (Wagner etal., 1982).] Reflectometer data show that the fluctuation decrease and the beginning of the $D_{\alpha}$ drop are coincident within $0.1 \mathrm{~ms}$. Within the $0.5 \mathrm{~ms}$ measurement accuracy of the spectroscopic system (Gohil etal., 1991b), the initial step in $E_{r}$ also occurs at the same time.

The time sequence of events at the $L$ to $H$ transition demonstrates that the change in $E_{r}$ is not a consequence of the change in edge profiles caused by the confinement improvement. As is illustrated in Fig. 3 , a substantial change in the electric field takes place prior to the time when the furst change occurs in the local gradients of jon temperature, carbon density, and carbon pressure. In othir words, the edge profiles have barely begun to change at a time when a significant $E_{r}$ is already estrablished. This sequence is what one would expect from a model in which the change in $E_{r}$ stabilizes fluctuations, which then leads to a change in the particle and heat fluxes. The usual conservation equations for particle and heat demonstrate that a change in the cross-field fluxes must ancumulate for a certain time before the radial profiles exhibit an appreciable response. A model in which the actual changes in the density and temperature profiles produce the change in $E_{\tau}$ is not consistent with the measured time bistory.

Although the initial, substantial change in $E_{r}$ seen in DIII-D precedes appreciable changes in the density and temperature profiles, both $E_{r}$ and those profiles can continue to evolve for many $10 s$ of milliseconds after the transition. This evolution has been noted on several tokamaks (Burrell et al., 1990; Field etal., 1991, 1992; Groebner et.al., 1990a, 1992; Ida et al., 1990, 1992).

Information on the causality question is also provided by the H-modes produced by plasma biasing in CCT (Taylor et al., 1989), TEXTOR (Van Nieuwentiove et al., 1991; Weynants et al., 1991) and TUMAN-3 (Askinazi et al., 1992). In these rnachines, the bias is applied first and $E_{r}$ in the plasma evolves to the poirit where the bifurcation occurs and the $\mathrm{H}$-mode develops. This sequence clearly shows the causal role of $E_{r}$. 

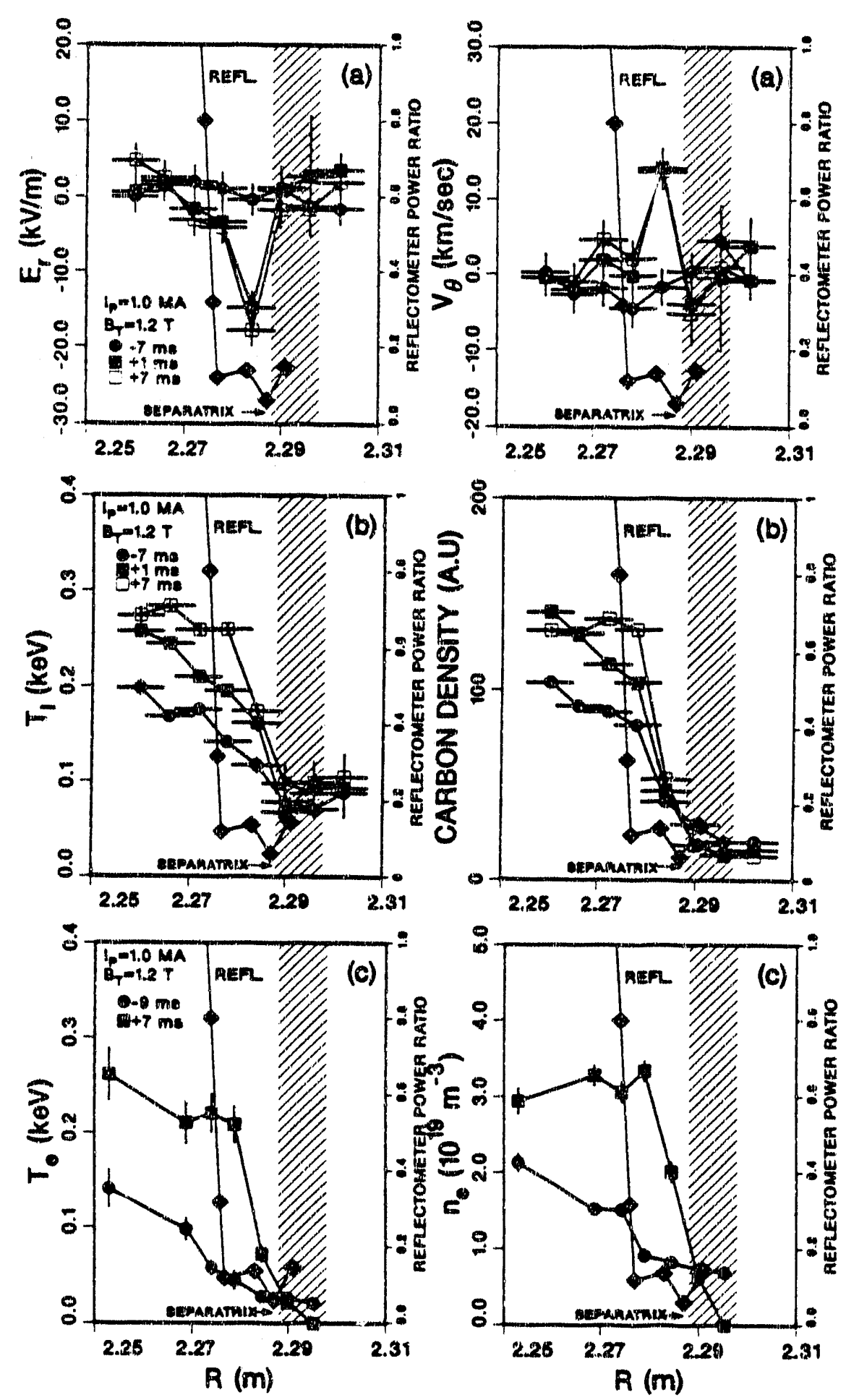

Fig. 1. Plots of various plasma profiles in DIll-D near the plasma edge at times relative to the $L$ to $H$ transition. 'The shaded region gives the best estimate of the separatrix location as determined from MHD equilibrium analysis. In addition to the profiles labeled on the left-hand vertical axis, each box contains a plot showing the change in the fluctuation power detected by the O-made and X-mode reflectometers (right and vertical scale). This is the ratio of the reflectometer power just, after the transition to that measured just before the transition. The reflectometer power is the amplitude of the signal integrated from $75 \mathrm{kHz}$ to the maximum detected frequency $(400$ to $800 \mathrm{kHz})$. The frequency integration is based on Fourier analysis over consecutive $0.1 \mathrm{~ms}$ intervals. (a) Profites of the poloidal rotation speed of $C$ VII and the radial electric field $E_{r}$ inferred from th. $L$ VII radial force balance equation as measured by charge exchange recombination spectroscopy. Integration time for the spectroscopic signal was 3 ms. (b) Profiles of the ion temperature and $C$ Vil density measured using the same technique as in (a). (c) Profiles of electron density and electron temperature measured by Thomson scattering. 


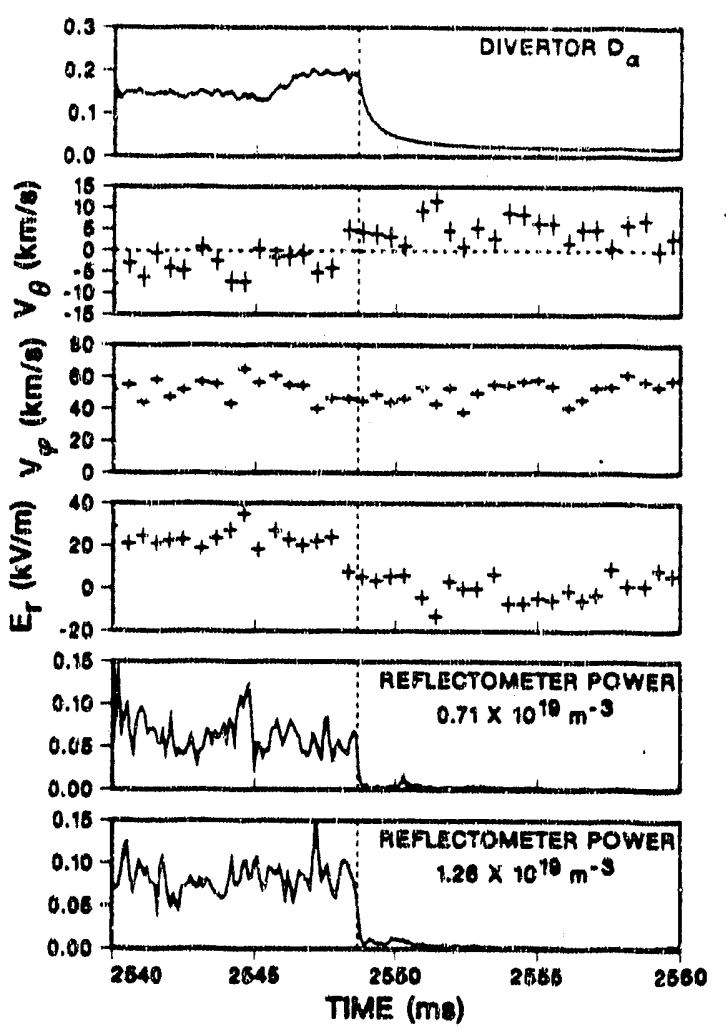

Fig. 2. Time history across the $L$ to $H$ transition in DIII-D of the divertor $D_{a}$ signal; edge poloidal rotation, toroidal rotation and inferred $E_{r}$ from charge exchange spectroscopy; and reflectorneter power. The $D_{a}$ signal and the reflectometer power have effective time resolutions of $0.1 \mathrm{~ms}$; the spectroscopic signals have a $0.5 \mathrm{~ms}$ integration time. The points for the spectroscopic results are plotted at a time which corresponds to the midpoint of the integration time interval. The spectroscopic signals come from the spatial location which corresponds to the minimum of $E_{r}$ [see Fig. 1(a)].

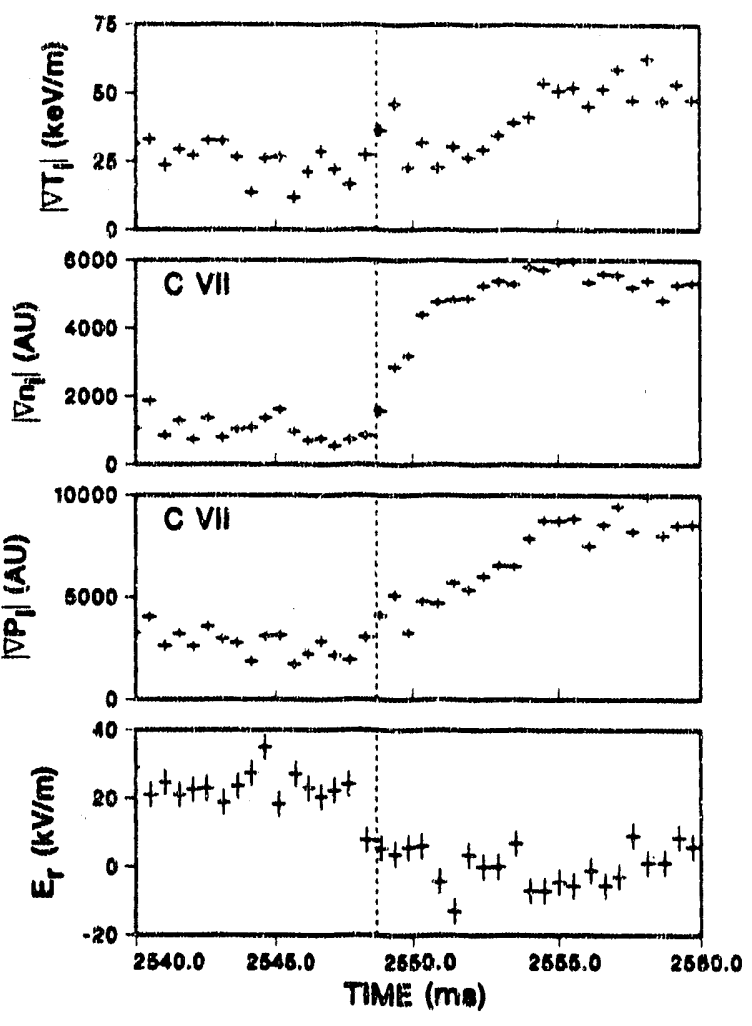

Fig. 3. Time history across the $L$ to $H$ transition in Dlll-D of edge ion temperature gradient, C VII density gradient, C VII pressure gradient and most negative edge $E_{r}$. The spatial location for all the curves is at the point just inside the separatrix where the most negative $E_{r}$ is seen and where, as shown in Fig. 1(a), the transport barrier forms. The negative jump in $E_{r}$ occurs prior to any' significant change in the edge ion gradients.

Theory predicts changes in the correlation lengths of fluctuations (Biglari et ai., 1990); changes consistent with theory have been seen in several machines. Radial and poloidal correla iion lengths of the turbulent fluctuations have been measured in the plasma edge by Langmuir probes in $C \mathrm{C} T$ (Taylor et al., 1991; Tynan et al., 1991), the radial coherence length has bee a determined by correlation reflectometry in DIII-D (Rhodes et al., 1991, 1992b), and poloidal correlation lengths have been inferred from FIR scattering (Rettig et al., 1992b) in DIII-D and from multipin Langmuir probe arrays in ASDEX (Niederrneyer et al., 1991). The Langmuir probe and reflectometer measurements both show a decrease in the radial coherence length by about a factor of two after the L to H transition. For example, in DIII-D, preliminary measurements of the the radial coherence length of density fluctuations in the plasma edge $(\rho \geq 0.98)$ show changes from roughly $0.4 \mathrm{~cm}$ in L-mode to $0.2 \mathrm{~cm}$ in $\mathrm{H}$-mode (Rhodes et al., 1991). (This work is still in a preliminary stage, since the radial coherence length only gives an upper limi: for the radial correlation length; a future publication will directly address this issue.) Qualitaively, this trend is what one would expect from the effect of sheared electric fields (Biglari etal, 1990). The poloidal correlation length, inferred from the $S\left(k_{\theta}\right)$ spectrum in DIII-D (taking account of $E \times B$ drifts), shows no significant change between L-mode and H-mode in DIII-D (Rettig et al., 1992b). In addition, in ASDEX, the $S\left(k_{\theta}, \omega\right)$ spectrum in H-mode shows no change except those due to differing $E \times B$ drifts when measured on either side of the separatrix (Niedermeyer et al., 1991). This lack of a change in the poloidal wavenumber spectrum is also what is predicted by theory for a measurement in the plasma frame. Poloidal correlation lengths in CCf measured in the laboratory frame increase substantially in H-mode (Taylor et al., 1991; Tynan, 1991). Transforming the theory to the laboratory frame, where the turbulent fluctuations are moving with the $E \times B$ velocity, should lead a substantial apparent increase in poloidal correlation length. Measurements of the turbulent decorrelation tirnes in a shear layer in an Ohmically heated limiter discharge (Ritz $\left.e^{\ddagger}, a l ., 1990\right)$ are also consistent with the theory. 
Because of diagnostic difficulties, there has been much less work on edge localized magnetic fluctuations across the $\mathrm{L}$ to $\mathrm{H}$ transition. As has been reported by (Burrell etal., 1989b, 1990; Malacarne etal., 1987; Matsumoto et al., 1992; Toi et al., 1989), there are significant decreases in both broadband magnetic fluctuations and in coherent MHD modes within a few $\mathrm{cm}$ of the separatrix. Recent theoretical calculations (Strauss, 1992) have indicated that sheared flow can stabilize drift tearing modes at the plasma edge. Accordingly, the magnetic fluctuation measurements are also consistent with the idea of electric field shear stabilization of turbulence.

\section{COMPARISONS WITH THEORY}

Theories of the $L$ to $H$ transition that are discussed in this section meet two criteria. First, since previous comparisons of theory and experiment have been done (Burrell et al., 1989b, 1990), new theoretical work had to be done since 1990 or new experinental evidence bearing on the theories had to be available before a theory would be included. Second, diagnostic measurements that could test key features of the theories had to be available. In some cases, potentially viable theories are not discussed because key quantities [e.g. turbulent Reynold's stress (Diamond and Kim, 1991)] could not yet be measured.

The work of (Biglari etal., 1990) does not discuss an $\mathrm{H}$-mode bifurcation condition as such but shows concisely how electric field shear can nonlinearly stabilize a broad class of general, flute-like turbulent modes in the plasma. The criterion for shear decorrelation to be important can be written

$$
\left|\frac{\nabla E_{r}}{\mathrm{~B}_{\mathrm{T}}}\right|>\frac{\Delta \omega_{t}}{k_{\theta} \Delta r_{t}}
$$

where $\mathrm{B}_{\mathrm{r}}$ is the toroidal field, $\Delta \omega_{t}$ is the turbuient decorrelation frequency, $\Delta r_{t}$ is the radial correlation length and $k_{\theta}$ is the poloidal wave number of the turbulence. $\Delta \omega_{t}$ and $\Delta r_{t}$ are the values in the absence of the electric field shear. Previous estimates have shown that the electric field shear measured in DIII- $D$ is sufficient for shear decorrelation to be important in the turbulence dynamics (Groebner etal., 1990a; Matsumoto etal., 1992). Using the profiles in Fig. 1, and typical values for $\Delta \omega_{t}=2 \pi \times 100 \mathrm{kHz}$ (Matsurnoto et al., 1992) and the radial coherence length of $0.4 \mathrm{~cm}$ as an estimate of $\Delta r_{i}$ (Rhodes et.al., 1991), shear decorrelation would be important for $k_{\theta} \geqslant 1 \mathrm{~cm}^{-1}$. FIR scattering results show the dominant fluctuation levels for this range of $k$-vectors.

An important feature of the theory by (Biglari et al., 1990) is that turbulence stabilization is independent of the sign of $\nabla E_{r}$. Accordingly, this theory can explain the H-mode with a positive hill structure in $E_{r}$ seen in TEXTOR biasing experirnents (Weynants et al., 1991) as well as the more usual, spontaneous $\mathrm{H}$-modes, which have a negative well in $E_{r}$.

As was discussed in the previous section, the theoretical predictions of how the radial and poloidal correlation lengths should change across the $\mathrm{L}$ to $\mathrm{H}$ transition agree with experimental measurements made at the outer (large major radius) midplane of the plasma. There are some measurements that indicate that behavior at the inner and outer midplane of the plasma is different. Previous work has shown that significantly more heat (Burrell et al., 1989b; Keilhacker et al., 1981) and pariicles (Tynan, 1991) flow through the large major radius side of the plasma in L-mode. In addition, heat flux measurements in the divertor (Burrell et al., 1989b) show changes across the L to $\mathrm{H}$ transition which are consistent with a marked decrease in heat flux through the outer major radius side of the plasma. Furthermore, radial correlation lengths measured near the inner midplane in CCT actually increase after the L to $\mathrm{H}$ transition ("Taylor et al., 1991).

The theory of (Biglari et al., 1990) can account for different behavior at the large and small major radius sides of the plasma if one considers the large variation in electric field shear caused by the tokamak's toroidal geometry. If we assume that the electrostatic potential is constant on a flux surface to lowest order, then the left hand side of Eq. (1) can be written as

$$
\left|\frac{\nabla E_{r}}{B_{\mathrm{T}}}\right|=\frac{\left(R B_{\mathrm{p}}\right)^{2}}{\left|\mathrm{~B}_{\mathrm{T}}\right|}\left|\frac{d^{2} \Phi}{d \psi^{2}}\right|
$$

where $\Phi$ is the electrustatic potential, $\psi$ the poloidal flux function (Hinton and Hazeltine, 1976), $\mathrm{B}_{\mathrm{p}}$ the poloidal field and $R$ the major redius. Since $d^{2} \Phi / d \psi^{2}$ is constant on a flux surface, the electric field shear term varies as $\left(R \mathrm{~B}_{\mathrm{p}}\right)^{2} / \mathrm{B}_{\mathrm{T}}$. This quantity is significantly smaller on the inner 
side of the plasma than the outer, varying crudely as $R^{3}$. The artual difference depends on the poloidal beta $\beta_{\mathrm{p}}$ of the plasma, with the value on the inner midplane approaching zero as $\beta_{\mathrm{p}}$ approaches the equilibrium limit. However, even for low $\beta_{p}$, typical values for DIII-D show that the $E_{r}$ shear term is at least a factor of 6 smaller at the inner midplane than at the outer. Utilizing the previous estimate, this would say that only fluctuations with $k_{\theta} \geq 6 \mathrm{~cm}^{-1}$ would be stabilized at the inner midplane. FIR scattering data show that most of the fluctuation power exists at smaller $k_{\theta}$. Accordingly, for a given electrostatic potential profile, stabilization of turbulence on the large major radius side should be much easier than on the small major radius side.

The work by Shäng and various colleagues (Shaing etal., 1989b, 1990, 1992a, 1992b) gives a clear bifurcation condition as well as a discussion of electric field shear stabilization of turbulence (Shaing et al., 1990). The theory has two main parts. First, a bifurcation in the main ion poloidal rotation causes a change in the radial electric field. Second, negative $E_{r}$ or more positive $\nabla E_{r}$ causes stabilization of turbulence and gives the confinement improvement (Shaing et al., 1990). A weaker $\mathrm{H}$-mode may be possible with positive $E_{r}$. The physical basis for the bifurcation is the need for the plasma to maintain zero net radial current in steady state. Accordingly, the nonambipolar radial current driven by poloidal rotation [Shaing et al., 1989b, 1990) is balanced by an extra radial current which can either be the directly driven current in biased plasmas, as in CCT, TEXTOR and TUMAN 3, or the current caused by loss of epithermal trapped particles scattering into the loss cone at the plasma edge. In the non-biased case, the ion collisionality $\nu_{\star i}$ near the plasma edge must approach unity for a significant loss of trapped, epithermal particles to occur. However, even for high $\nu_{x i}$, multiple solutions exist for the plasma rotation near the edge. In the absence of any other force, the need for significant ion orbit loss gives $\nu_{\text {ti }}$ near unity as an approximate bifurcation condition. This condition on $\nu_{m i}$ is unimportant if the driving current is provided by another means. In addition, if ion orbit loss is important, the width of the ion banana orbit at the plasma edge sets the scale length for the radial electric field (Shaing, 1992a).

The general picture of the development of the H-mode edge shown in Figs. 1 through 3 is in agreement with Shaing's theory. In addition, the form of the relationship between the radial current and radial electric fiwld seen in biased TEXTOR plasmas (Weynarits et al., 1991) agrees with the theory. Furthermore, the size of the shear layer at the plasma edge depends weakly on plasma current (Doyle et al., 1991; Groebner et al., 1991), in agreement with the theory including the effects of shear in the radial electric field on the banana orbit width (Shaing, 1992a).

In all spontaneously triggered $\mathrm{H}$-modes studied to date, $E_{r}$ is negative near the plasma edge (Burrell et al., 1990; Doyle et al., 1991; Field et al., 1991, 1992; Gohil et al., 1991a, 1991b; Groebner et al., 1990a, 1991, 1991; Ida etal., 1990, 1992). This is in accord with the stated prediction of the theory. However, the simple approximation $E_{r}=$ constant which is used in the basic stability derivation (Shaing et al., 1990) is clearly too crude; where measurements exist of the electric field structure, (Doyle et al., 1991; Gohil et al., 1991a, 1991b; Groebner et al., 1990b, 1991; Ida et al., 1990, 1992; Van Nieuwenhove etal., 1991), they show a very rapid variation in $E_{r}$ near but inside the separatrix. This assumption of constant $E_{r}$ in the primary stability calculation may be one reason why the publication (Shaing et al., 1990) states turbulence stabilization should occur primarily for for $\nabla E_{r}>0$ even though the fundamental equations are independent of the sign of the poloidal shearing rate (Shaing et al., 1990). If the poloidal shearing is dominated by $\nabla E_{r}$, then, as discussed by (Biglari et al., 1990), turbulence stabilization for either sign of $\nabla E_{r}$ should be possible. Such a prediction would be in accord with the experiments, which see negative well in $E_{r}$ in spontaneous $\mathrm{H}$-mode (Doyle et al., 1991; Gohil et al., 1991a; Groebner et al., 1990a, 1991; Ida et al., 1990, 1992) and both signs of $E_{r}$ in biased H-mode (Taylor et al., 1989, 1991; Van Nieuwenhove et al., 1991; Weynants et al., 1991).

The published theory has $\nu_{\star i}$ around or.e as an approximate bifurcation condition. In addition, the theory predicts (Shaing et al., 1989, 1990) that the normalized poloidal rotation parameter

$$
U_{\mathrm{pm}}=\frac{1}{v_{\mathrm{th}} \mathrm{B}_{\mathrm{p}}}\left[v_{\theta} \mathrm{B}_{\mathrm{T}}-\left(\frac{1}{Z_{i} e n_{i}}\right) \nabla \mathrm{p}_{i}\right],
$$

should be significantly below unity in L-roode and above unity in $\mathrm{H}$-mode. Here, $v_{\theta}$ is the poloidal rotation speed of the main ions, $\tau_{i}$ is the ion density, $Z_{i}$ is the ion charge, $p_{i}$ is the ion pressure and $v_{\text {th }}=\left(2 T_{i} / m_{i}\right)^{1 / 2}$ is the ion thermal speed. As is shown in Fig. 4 , the theoretically expected values of $\nu_{* i}$ and $U_{\mathrm{pm}}$ agree with what is seen in DIII-D. The measurements of $U_{\mathrm{prn}}$ shown in Fig. 4 are the first in which this quantity has been computed by directly measuring the poloidal rotation and pressure gradient for the rain (helium) ions. Previous measurements of associated quantities (Ida etal., 1990, 1992) have used the poloidal rotation speed of impurity ions. The theoretical expectations for of $\nu_{m i}$ are not born out in JFT2-M (Ida et al., 1990, 1992). There L to $\mathrm{H}$ transitions are seen in plasmas which have $\nu_{m i}$ around 50 in both $\mathrm{L}$-mode and $\mathrm{H}$-mode. 


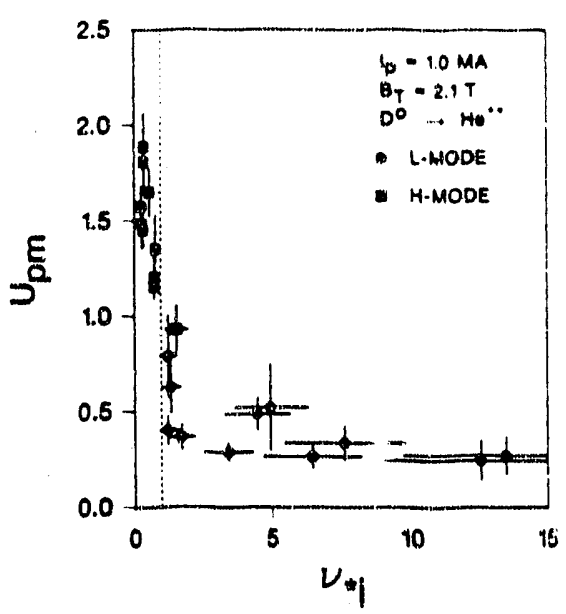

Fig. 4. Normalized poloidal rotation parameter for main plasma ions $U_{\mathrm{pm}}$ from Eq. (3) plotted as a function of $\nu_{\star i}$ for helium plasmas in DIII-D with $I_{\mathrm{p}}=1.0 \mathrm{MA}, \mathrm{B}_{\mathrm{T}}=2.1 \mathrm{~T}$ and with a time-averaged deuterium neu. trai injection power of $4.8 \mathrm{MWV}$. "These plasinas are single-null divertor plasmas with the ion $\nabla B$ drift towards the $X$-point. The general geometry definition of $\nu_{\text {ari }}$ used is given in (Kim, 1991) and does not include the effect of impurityminn collisions. The helium ion density needed in eval. wating $\nu_{* i}$ is determined from charge exchange recombination spectroscopy.

One of the problems in matching the theoretical bifurcation condition $\nu_{* i} \simeq 1$ with the experimental measurements is the lack of a proper definition for $\nu_{\star i}$ for a finite aspect ratio divertor plasma with multiple jon species. The standard definition $\nu_{\star i}=\nu_{i i} R q / \epsilon^{i / 2} v_{t h}$ is for a large aspect ratio limiter plasma with one ion species. This definition is, unfortunately, singular on the separatrix of a divertor plasma. The JFT2.M work (Ida et al., 1990, 1992) utilizes this definition but evaluates it $0.7 \mathrm{~cm}$ inside the separatrix. The DIII-D work shown in Fig. 4 uses a general geometry definition for $\nu_{* i}$ (Kim etal., 1991b) which also does not include imapurity effects.

The work of the Itohs $(1988,1989 a, 1989 b, 1990)$ has been through several iterations. The latest version (Itoh and Itoh, 1990) includes the effects of ion orbit loss, similar to the Shaing model, but also includes the possibility of anomalous electron loss. This electron loss takes the place of the viscosity driven radial current in Shaing's model. A nonambipolar loss in either the electron or ion channel (or both) is required to balance the loss of banana trapped particles at the plasma edge, thus maintaining zero net radial current in steady state. The latest version of both models (Itoh and Itoh, 1990; Shaing, 1992a) include the effects of $\nabla E_{r}$ in squeezing the ion orbits, as wras first discussed by Hazeltine (1989). Unfortunately, because of the form that they employ for the ion orbit loss, the Itoh's model is really only applicable when the plasma edge is in the extreme banana regime, $\nu_{\star i} \ll 1$. At the time of the $L$ to $\mathrm{H}$ transition, the edge of the plasma in most tokamaks has $\nu_{m i} \geqq 1$ (Burrell et al., 1989b; Ida et al., 1990, 1992; Wagner et al., 1985); this difference renders quantitative comparisons with the theory moot. The quantitative comparisons that have been done for JFT2-M show reasonable agreement for the bifurcation condition and for the electric

field shear (Ida et al., 1990, 1992). The agreement with the DIII-D data is not as good. For the data in Fig. 1, the bifurcation parameter

$$
\lambda=-\left(\frac{T_{e}}{T_{i}}\right) \rho_{\mathrm{pi}}\left(\frac{\nabla n_{e}}{n_{e}}+\frac{\alpha \nabla T_{e}}{T_{e}}\right)
$$

does change from $0.8 \mathrm{in} \mathrm{L-mode} \mathrm{to} 2.7 \mathrm{in} \mathrm{H}$-mode, in agreement with the theoretical boundary ait $\lambda \simeq 1$. Here, $\rho_{\mathrm{pi}}$ is the poloidal ion gyro-radius. However, the electric field shear parameter $u_{g}=\left(\rho_{p i} / v_{t i} \mathrm{~B}_{\mathrm{p}}\right) \nabla E_{\mathrm{r}}$ is zero in L-mode and about -0.5 in $\mathrm{H}$-mode; this disagrees with a theoretical $\mathrm{L}$-mode value of +1 and $\mathrm{H}$-mode value of -2 . The theory is capable of having either sign of $E_{r}$ in the $\mathrm{H}$-mode; however, to obtain $E_{r}<0$ requires $\left|\nabla T_{i} / T_{i}\right| \geq \mid 5.9 \nabla n_{i} / n_{i}$. [The numerical value 5.9 comes from the relationship between foroidal rotation speed and $\nabla t_{i}$ given by (Hazeltine, 1974).] This large a $T_{i}$ gradient is not seen in present experiments. Thus, the theory would predict positive electric fields in most cases, in disagreement with win.. is seen experimentally. The Itoh's model has been extended from a point model to a one dimensional model to confront edge localized modes (ELMs) (Itoh etal., 1991); use of the one dimensional equations to confront the basic transition would perhaps lead to a better match with experirnent.

A theory to explain the $L$ to $H$ transition based on a bifurcation in the ion heat transport equation has been proposed by Hinton (1991). This model has several functional dependences which agree with experimental results. For example, the model has an $\mathrm{H}$-mode power threshold which increases with density and toroidal field, in agreement with experiment (Burrell et al., 1989a; Carlstrom, 1989). In addition, the theory predicts a lower density limit for the H-mode transition, in agreement with ASDEX measurements (Wagner et al., 1982). However, although the theory predicts a steep temperature gradient region inside the separatrix, it would also predict a rapid variation of the width of this region with input power, which is not seen experimentally. This lack of dependence on input power is shown in Fig. 5. The problem with the existing version of the theory is that the transport coefficients are taken to be constant. Because the theory has a bifurcation character, there is a minimum heat flux required for the ion heat transport equation to stay on the steep gradient branch. The constant transport coefficients cause this minimum to also be constant. Accordingly, if the input power is increased substantially (e.g. by a factor of four as 

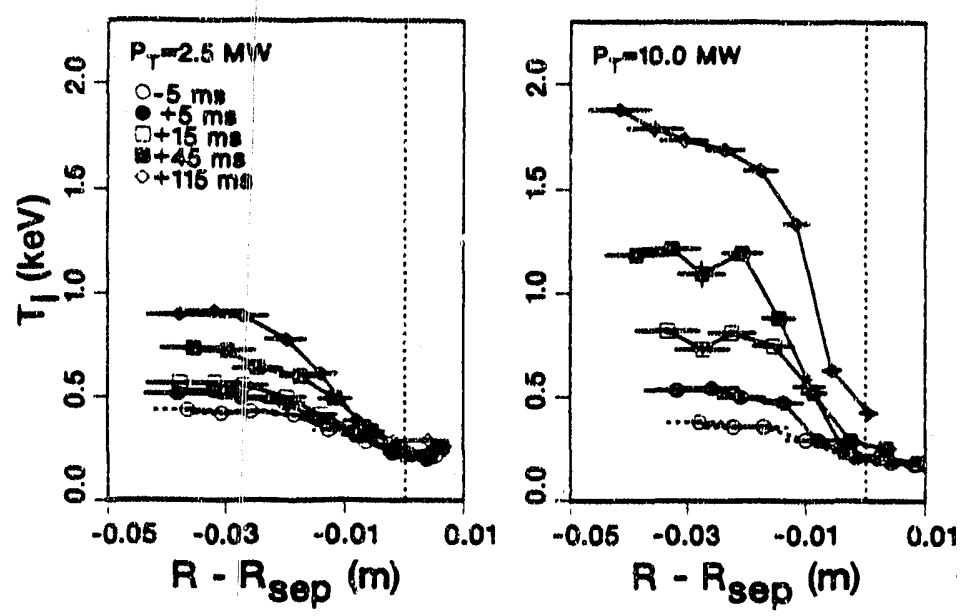

Fig. 5. Tims sequence of ion temperature profiles near the plasma edge discharges with $2.5 \mathrm{MW}$ and $10 \mathrm{MW}$ input neutral beam power. Basic discharges conditions are single-null divertor, $Y_{P}=1.0 \mathrm{MA}, \mathrm{B}_{\mathrm{T}}=2.1 \mathrm{~T}$, minor radius $0.65 \mathrm{~m}$, and vertical ulongation of 1.9. Times in the graphs are given relative to the $L$ to $H$ iransition. Positions are plotted relative to the separantrix location determined from MHD equilibrium analysis. Not:e the width of the region of steep gradients does not increase from the low power to the high power case.

in Fig. 5), then the position in normalized minor radius $r / a$ where the heat flux matches the minimum decreases by at least the same factor and possibly much more, depending on the actual heat flux profile. For example, if the lower heat flux had the boundary of the steep gradient region at $r / a=0.95$, then a factor of four increase would place the boundary insicie of $r / a=0.24$. A steep gradient region that extends this far into the plasma is inconsistent with Fig. 5 and has never been seen in any $\mathrm{H}_{\mathrm{i}}$-mode experiment. Although the model has several functional dependence that match experiment, the constant transport coefficient assumption requires improvement.

As rnentioned previously, there are theories for which the crucial diagnostic measurements are beyond the state of the current diagnostic art. For example, the turbulent Reynolds stress needed by Diamond and Kim (1991) has not H-mode; the radial gradient of the poloidally asymmetric portion of the radial particle flux required by Hassam et al., (1991a) is similarly inaccessible at present. Both of these quantities can probably be measured using Langmuir probes; however, the poloidally distributed measurements required by the latter will be a technological challenge. Both of these theories are based on the idea that the (turbulent) flows in the plasna edge are unstable to the creation of significant sheared $E \times B$ flow. However, the plasma in the $\mathrm{H}$-mode appears to be much less turbulent and much more poloidaliy symmetric than the I-mode. The fundamental question then is: How does the plasma maintain the more quiescent, more symmetric $\mathrm{H}$-mode state when the driving terms from turbulence or poloidal asymmetry that existed in L-mode have disappeared? When plasma heating is turned off, or when the plasma bias voltage is turned off, tokin.lak plasmas always go back to $\mathrm{L}$-mode. Accordingly, the $\mathrm{H}$-mode state requires a certain driving term to maintain it. These theories of spontaneously excited spin-up need to identify the driving terms and specify how they continue to exist in $\mathrm{H}$-mode.

\section{CONCLUSIONS}

Combined theoretical and experimental work has resulted in the the creation of a paradigm which has allowed qualitative understanding of many features of the $\mathrm{H}$-mode. Shear in the $E \times B$ flow of the fluctuations in the plasma edge can lead to decorrelation of the fluctuations, decreassd radial correlation lengths and reduced turbulent transport. Behavior consistent with effects of shear in $E_{r}$ have been seen in several tokamaks. At the L to $H$ transition, the electric field shear develops in a localized region near the plasma edge. Turbulent density fluctuations decrease in the same region. Particle and energy transport decrease there also, as indicated by the increase in the local gradients and by direct measurements of the local, fluctuation-driven particle flux. In addition, a significant change in the electric field occurs right at the transition, before the radial profiles of density and temperature have had a chance to change appreciably. This indicates that the initial change in $E_{r}$ is not caused by the improvement in confinement altering the radial profiles of density and ternperature, although $E_{r}$ may change further while the profiles continue to evolve. Furthermore, the radial correlation length of the density fluctuations decreases at the transition while the poloidal correlation length remains unchanged, as is expected from theory. Finally, experiments with $\mathrm{H}$-modes produced by direct biasing of the plasma provide further information on causality by showing that altering the radial electric field causes the transition to occlir.

Although we now have a basic model which allows us to understand the confinement ir.zprovement that occurs at the $\mathrm{L}$ to $\mathrm{H}$ transition, we are still lacking a well tested theory of how the crucial electric field shear is created. Producing and testing this model is the next challenge in $\mathrm{L}$ to $\mathrm{H}$ transition physics. Since the turbulen ze stabilization theories (Biglari etal, 1990; Shaing etal., 1990 ) indicate that the radial structure of $E_{r}$ is important in stabilizing turbulence, we need to move from theories which a consider only a point in space to theories that are at least one dimensional, so that $E_{r}(r)$ and, hence, $\nabla E_{r}$ can be computed. Testing the various theories will require continuing diagnostic improvernent. 
The authors would like to acknowledge fruitful discussions with P.H. Diamond, A.R. Field, G. Fussmann, F.L. Hinton, K. Ida, S.-I. and K. Itoh, Y.B. Kim, T.K. Kurki-Suonio, K.C. Shaing, R.J. Taylor, G. Tynan and F. Wagner. This work was supported by the U.S. Department of Energy under Contract Nos. DE-AC03-89ER51114, DE-FG03-89ER51121, DE-FG03-86ER53225, DE-AC05-84OR21400, and DE-AC03-76DP00789.

\section{REFERENCES}

Askinazi, L. G., V. E. Golant, S. V. Lebedev, etal., (1932). Nucl. Fusion 32, 271.

Biglari, H., P. H. Diamond, and P. W. Terry (1990). Phys. Fluids B2, 1.

Burns, S. R., T. L. Rhodes, W. A. Peebles, etal., (1991). Bull. Am. Phys. Soc. 36, 2499.

Burrell, K. H., S. L. Allen, G. Bramson, et al., (1989a). Plasma Physic and Controlled Nuclear Fusion Research 1988 (International Atomic Energy Agency, Vienna, 1989), Vol. 1, p. 193.

Burrell, K. H., S. L. Allen, G. Bramson, et al., (1989b). Plasma Physics and Controlled Fusion 31, 1649 .

Burrell, K. H., T. N. Carlstrom, E. J. Doyle, etal., (1990). Phys. Fluids B2, 1405.

Carlstrom, T. N., M. Shimada, K. H. Burrell, et al., (1989). Proc. 16th European Conf. ori Controlled Fusion and Plasma Physics, Venice, Vol. 13B,, 241.

Carlstrom, T. N., G. L. Campbell, J. C. DeBoo, et al., (1996). Rev. Sci. Instrum. 61, 3016.

Carreras, B. A., K. Sidikman, P. H. Diamond et al., (1992), ORNL report P-91/51278, submitted to Phys. Fluids $B$.

Costley, A. E., P. Cripwell, R. Prentice, and A. C. C. Sips (1990), Rev. Sci. Instrum. 61, 2823.

Cripwell, P., A. E. Costley, and A. E. Hubbard (1989), Proc. 16th European Conf. on Controlled Fusion and Plasma Physics, Venice, Vol. 13B, Part I p. 75.

Diamond, P. H., and Y. B. Kim (1991), Phys. Fluids B 3, 1626.

Dodel, G., E. Holzhauer, H. Niedermeyer, et al., (1991). Proc. 18th European Conf. on Controlled Fusion and Plasma Physics, Berlin Vol. 15C, Part I, p. 273.

Dodel, G., E. Holzhauer, and the ASDEX Team (1992). Proc. 19th European Conf. on Controlled Fusion and Plasma Physics, Innsbruck (to be published).

Doyle, E.J., T. Lehecka, N. C. Luhmann,. Jr., et al., (1990), Rev. Sci. Instrum. 61, 3016.

Doyle, E. J., R. J. Groebner, K. H. Burrell, et al., (1991). Phys. Fluids B3, 2300.

Field, A. R., G. Fussmann, J. V. Hofmann (1991). Proc. 18th European Conf. on Controlled Fusion and Plasma Physics, Berlin, Vol. 15C, Part III, p. 113.

Field, A. R., G. Fussmann, J. V. Hofmann (1992). Nucl. Fusion (to be published).

Gohil, P., K. H. Burrell, R. J. Groebner, and R. P. Seraydarian (1990). Rev. Sci. Instrum. 61, 2949.

Gohil, P., K. H. Burrell, E. J. Doyle, et al., (1991a). Proc. 18th European Conf. on Controlled Fusion and Plasma Physics, Berlin, Vol. 15C, Part I, p. 289.

Gohil, P., K. H. Burrell, R. J. Groebner, et al., (1991b). Proc. 14 th IEEE/NPSS Symposium on Fusion Technoloyy, Vol. II, p. 1199.

Groebner, R. J., P. Gohil, K. H. Burrell, T. H. Osborne, R. P. Seraydarian, H. St. John (1989). Proc. 16th European Conf. on Controlled Fusion and Plasma Physics, Venice, Vol. 13B, Part I, P. 245.

Groebner, R. J., K. H. Burrell, and R. P. Seraydarian (1990a). Phys. Rev. Lett. 64, 3015.

Groebner, R. J., K. H. Burrell, P. Gohil, and R. P. Seraydarian (1990b). Rev. Sci. Irustrum. 61, 2920 .

Groebner, R. J., W. A. Peebles, K. H. Burrell, etal., (1991). Plasma Phusics and Controlled Nuclear Fusion Research 1990 (International Atomic Energy Agency, Vienna, 1991) Vol. 1, p. 453.

Groebrer, R. J., K. H. Burrell, P. Gohil, J. Kim, and R. P. Seraydarian (1992). Proc. 19th European Conf. on Controlled Fusion and Plasma Physics, Innsbruck Vol. 16C, Part I, p. 183.

Hassam, A. B., T. M. Antonsen, Jr., J. F. Drake, and C. S. Liu (1991a). Phys. Rev. Lett. 6., 309.

Hassam, A. B. (1991b). Comments Plasma Phys. Controlled Fusion 14, 275.

Hawkes, N., et cl., (1992). Rev. Sci. Instrum. (to be published)

Hazeltine, R. D (1974). Phys. Fluids 17, 961.

Hazeltine, R. D (1989). Phys. Fluids B1, 2031.

Hinton, F.L., and R. D. Hazeltine (1976). Rev. Mod. Phys. 48, 239, Eq. (2.45).

Hinton, F. L., (1985). Nucl. Fusion 25, 1457.

Hinton, F. L. (1991). Phys. Flzids B3, 696.

Holzhauer, E., G. Dodel, and the ASDEX Tearn (1990). Rev. Sci. Instrum. 61, 2817.

Ida, K., and S. Hidekuma (1989). Rev. Sci. Instrum. 60, 867.

Ida, K., S. Hidekuma, Y. Miura, et cl., (1990). Phys. Rev. Lett. 65, 1364.

Ida, K., S. Hiidekuma, M. Kojima, et al., (1992). Phys. Fluids B4 (to be published).

Itoh, S.-I., and K. Itoh (1988). Phys. Rev. Lett. 60, 2276.

Itoh, S.-I., K. Itoh, T. Ohkawa, etal., (1989a). Plasma Physics and Controlled Nuclear Fusion Research 1988 (International Atomic Energy Agency, Vienna, 1989) Vol. 2, p. 23. 
Itoh, S.-I., and K. Itoh (1989b). Nucl. Fusion 29, 1031.

Itoh, S.-I., and K. Itoh (1990). J. Phys. Soc. Japan 59, 3815.

Itoh, S.-I., K. Itoh, A. Fukuyama, Y. Miura, etal., (1991). Phys. Rev. Lett. 67, 2485.

Jacksor, G. L., J. Winter, T. S. Taylor, et al., (1992). Phys. Fluids B4 (to be published).

Keilhacker, M., and the ASDEX Team (1981). Proc. IAEA Technical Committee Meeting on Divertors and Impurity Controh, M. Keilhacker and U. Daybelge, Ed. (Garching, Germany), 23.

Kim, Y. B., P.H. Diamond, H. Bigiari, and J. D. Callen (1991a). Phys. Fluids B3, 384.

Kim, Y. B., P. H. Diamond, and R. J. Groebner (1991b). Phys. Fluids B3, 2050.

Kim, Y. J., K. W. Gentle, Ch. P. Ritz, T. L. Rhodes, and R. D. Bengston (1991c). Phys. Fluids B3, 674 .

Kurki-Suonio, T. K., R. J. Groebner, K. H. Burrell (1992). Nucl. Fusion 32, 133.

Lehecka, T., etal., (1988). Rev. Sci. Instrum. 59, 1620.

Malacarne, M., P. Cripwell, P. A. Duperrex, et al., (1987). Plasma Physics and Controlled Fusion 281675.

Manso, M. E., F. Wagner, J. Matias, et al., (1991). Proc. 18th European Conf. on Controlled Fusion and Plasma Physics, Berlin, Vol. 15C, Part I, p. 393.

Matsumoto, H., K. H. Burrell, T.N. Ca-lstrom, etal., (1992). Plasma Physics and Controlled Fusion 34,615.

Moyer, R. A., J. G. Watkins, R. W. Conn, etal., (1992). " $E \times B$ Transport in the DIII-D Boundary Plasma," University of California Report UCLA-PPG-1433, to be published in J. Nucl. Mater.

Niedermeyer, H., A. Carlson, M. Endler, et al., (1991). Proc. 18th European Conf. on Controlled Fusion and Plasma Physics, Berlin, Vol, 15C, Part I, p. 301.

Ohkawa, T., F.L. Hinton (1987). Plasma Physics and Controlled Nuclear Fusion Research 1986 (Iniernational Atomic Ene, gy Agency, Vienna, 1987) Vol. 2, p. 221.

Peebles, W. A., S. Baang, D. L. Brower, et al., (1990). Rev. Sci. Instrum. 61, 3509.

Philipona, R., E. J. Doyle, N. C. Lubmann, Jr., et al., (1990). Rev. Sci. Instrurn. 61, 3007

Philipona, R., R. J. Groebner, K. H. Burrell, et al., (1991a). "Two-Step Turbulence Suppression and $E \times B$ Plasma Flow Measured at the L-H Transition," General Atomics Report GAA20516, submitted to Phys. Fluids.

Philipona, R., E. J. Doyle, N. C. Luhmann, Jr., et al., (1991b). Proc. 18th European Conf. on Controlled Fusion and Plasma Physics, Berlin, Vol. 15C, Part I, p. 281.

Rettig, C. L., S. Burns, R. Philipona, et al., (1990). Rev. Sci. Instrum. 61, 3010.

Rettig, C. L., W. A. Peebles, K. H. Burrell, et al., (1992a). Proc. 1.9t/2 European Conf. on Controlled Fusion and Plasma Physics, Innsbruck Vol. 16C, Part I, p. 179.

Rettig, C. L., W. A. Peebles, K. H. Burrell, et al., (1992b). Submitted to Nucl. Fusion.

Ritz, Ch. P., H. Lin, T. L. Rhodes, and A. J. Wooten (1990) Phys. Rev. Lett. 65, 2543.

Rhodes, T. I., W. A. Peebles, E. J. Doyle, and C. Rettig (1991). Bull. Am. Phys. Soc. 36, 2474.

Rhodes, T. L., S. Baang, A. E. Chou, C. W. Domier, N. C. Luhmann, Jr. (1992a). Rev. Sci. Instrum. (to be published).

Rhodes, T. L., W. A. Peebles, and E. J. Doyle (1992b). Rev. Sci. Instrum. (to be published).

Shaing, K. C., G. S. Lee, B. A. Carreras, W. A. Houlberg, E. C. Crume, Jr. (1989a). Plasma Physics and Controlled Nuclear Fusion Research 1988 (International Atomic Energy Agency, Vienna, 1989) Vol. 2, p. 13.

Shaing, K. C., and E. C. Crume, Jr. (1989b). Thys. Rev. Lett. 63, 2369.

Shaing, K. C., E. C. Crume, Jr., and W. A. Houlberg (1990). Phys. Fluids B2, 1492.

Shaing, K. C., (1992a). Phys. Fluids B4, 290.

Shaing, K. C., R. D. Hazeltine, H. Sanuki (1992b). Phys. Fluids B4, 404.

Staebler, G. M., and R. R. Dominguez (1991). Nucl. Fusion 31, 1891.

Strauss, H. R., (1992). Phys. Fluids B4, 3.

Taylor, R. J., M. L. Brown, B. D. Fried, et al., (1989). Phys. Rev. Lett. 63, 2365.

Taylor, K. J., R. W. Conn, B. D. Fried, et al., (1991). Plasma Physics and Controlled Nuclear Fusion Research 1990 (International Atomic Energy Agency, Vienna, 1990) Vol. 1, p. 463.

'Toi, K., J. Gernhardt, O. Klueber, and M. Kornherr (1989). Phys. Rev. Lett. 62, 430.

Tynan, G. R., (1991). PhD Thesis, University of California Los Angeles Report UCLA-PPG-1369.

Tynan, G. R., L. Schmitz, R. W. Conn, etal., (1992). Phys. Rev. Lett. 68, 3032.

Van Nieuwenhove, R., G. Van Oost, R. R. Weynants, etal., (1991). Froc. 18th European Conf. on Coritrolled Fusion and Plasma Physics, Berlin, Vol. 15C, Part I, p. 405.

Wagner, F., G. Becker, K. Behringer, et al., (1982). Phys. Rev. Lett. 49, 1408.

Wagner, F., R. Bartiromo, G. Becker, et al., (1985). Nucl. Fusion 25, 1490.

Wagner, F., F. Ryter, A. R. Field, et al., (1991). Plasma Physics and Controlled Nuclear Fusion Research 1990 (International Atomic Energy Agency, Vienna, 1991), Vol. 1, p. 277.

Weynants, R. R, D. Bora, T. Delvigne, etal., (1991). Plasma Physics and Controlled Nuclear Fusion Research 1990 (International Atomic Energy Agency, Vienna, 1991), Vol. 1, p. 473. 

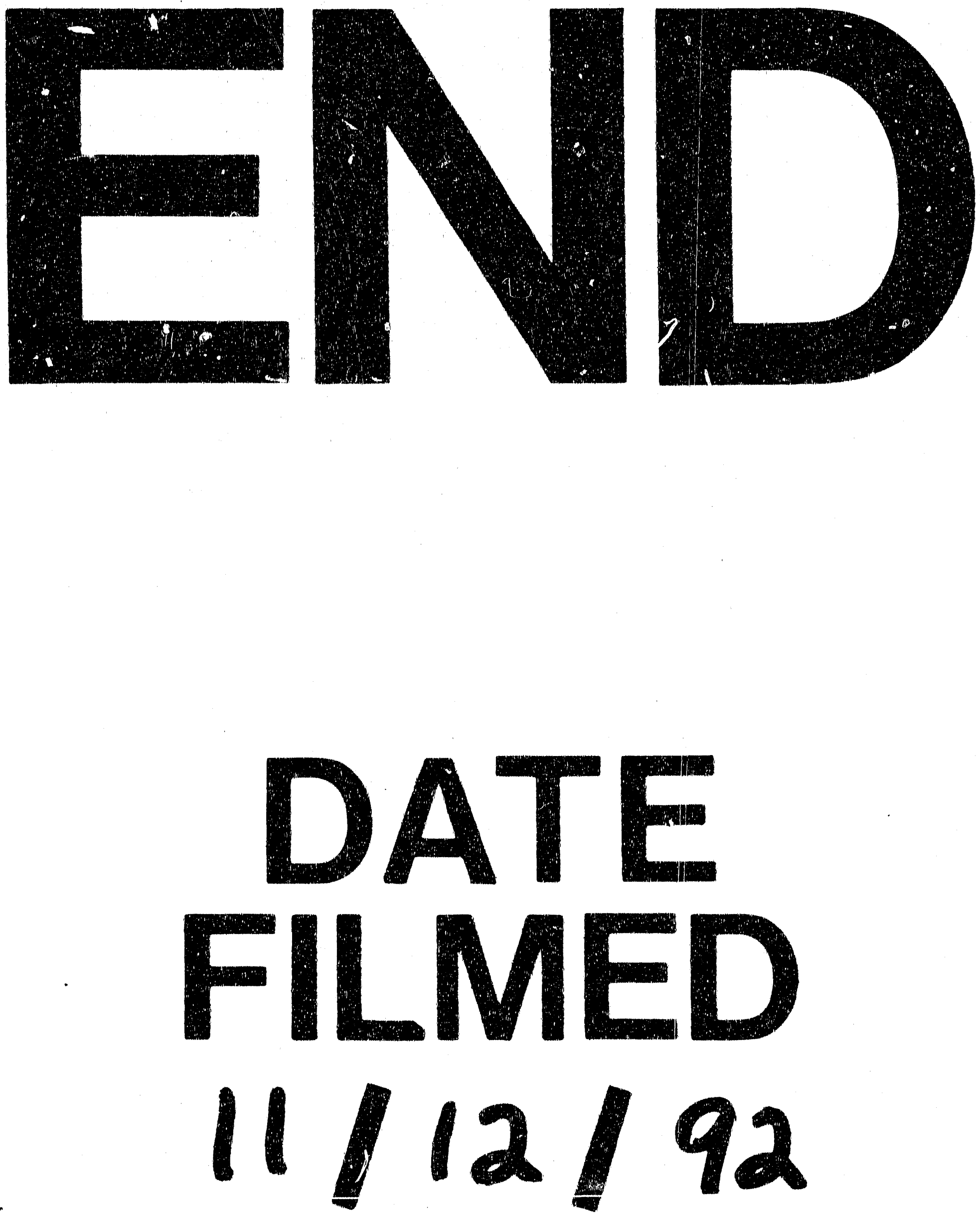
